Acta Horticulturae et Regiotecturae 2

Nitra, Slovaca Universitas Agriculturae Nitriae, 2019, pp. 56-60

\title{
DIFFERENCES IN WATER VAPOR ADSORPTION-DESORPTION OF NON AGED AND 3-YEAR AGED BIOCHAR IN SANDY SPODOSOLS
}

\author{
Eugene BALASHOV*, Irina MUKHINA, Elena RIZHIYA \\ Agrophysical Research Institute, St. Petersburg, Russia
}

\begin{abstract}
Ageing of biochars in soil affects their surface properties and can cause changes in water vapor adsorption-desorption processes. Measurements of hygroscopic water contents and corresponding water potentials of non aged and 3-year aged biochars as well as of sandy soils with medium and high quality were carried out during 5 cycles of water vapor adsorption-desorption processes at a room temperature of $23.5^{\circ} \mathrm{C}$. The results showed a significantly lower content of maximum hygroscopic water in the aged biochars than that in the non aged biochar at the end of water vapor adsorption processes at high air humidity. A significantly higher affinity of the high quality soil to water vapor resulted in insignificant differences in the maximum hygroscopic water content and in significant changes in the corresponding water potentials as compared to the same properties of the soil with medium quality. Minimum content of hygroscopic water was significantly lower in the non aged biochar than in the aged biochars at the end of the water desorption processes at ambient laboratory atmosphere. There were insignificant differences in minimum contents of hygroscopic water and in the corresponding water potentials of the aged biochars from soils with medium and high quality.
\end{abstract}

Keywords: biochar, ageing, water vapor adsorption-desorption

In current conditions of climate change soils can be subjected to impacts of extreme weather factors and anthropogenic treatments which can lead to unfavorable changes in the indicators of their quality and sustainability. Apart from rational application of organic and mineral fertilizers, agricultural use of biochar is a promising way of sustainable and environmental management of soils (Lehmann et al., 2011).

Biochar is a product of the incomplete combustion of agricultural and forest waste. Organic matter of biochar includes amorphous and crystalline aromatic structures and, therefore, is resistant to biotic and abiotic mineralization (Keiluweit et al., 2010). Biochar is being produced in the process of slow (hours) and fast (minutes) pyrolysis of biomass without access of oxygen at high temperatures of $300-900{ }^{\circ} \mathrm{C}$. Carbon content in biochar can exceed $80 \%$ depending on technological conditions of pyrolysis and feedstock type (Lehmann et al., 2011). Technological conditions of pyrolysis and type of feedstock result in different properties of biochar.

Incorporation of biochar into arable soils can lead to enhancing carbon sequestration, improvement of physical, physico-chemical, biological soil properties and to decreasing $\mathrm{N}_{2} \mathrm{O}$ emission from soils (El-Naggar et al., 2019; Horák, 2015).

The recalcitrant nature of biochar does not mean that it remains unchanged when ageing in soils. The quantification of changes in the surface properties of biochar while ageing in soils is a crucial aim of current research. An oxidation of biochar in soils can lead to an increase in a density of oxygen-containing functional groups (OFGs) on its surface. The high density of polar OFGs (carboxyl, carbonyl and phenolic hydroxyl) on the biochar surface can increase its affinity to water molecules, nutrients and organic pollutants.

Current hydrophysical studies of biochars are mainly focused on measurements of their water retention curves and available water contents at high matric potentials (>-15 MPa) using pressure plate chambers (Marshall et al., 2019). Less attention has been paid to the assessment of water vapor adsorption-desorption characteristics of biochar surface while ageing in soils. Scientific information on these processes is needed for better understanding of temporal changes in the surface structural properties of aged biochars.

The objective of this study was to quantify the changes in water vapor adsorption-desorption characteristics of biochar after 3-year ageing in sandy Spodosols with medium and high quality.

\section{Material and methods}

The study was carried out at the Menkovo experimental station of the Agrophysical Research Institute in the St. Petersburg region of Russia $\left(59^{\circ} 34^{\prime} \mathrm{N}, 30^{\circ} 08^{\prime} \mathrm{E}\right)$. The studied soil was classified as sandy Spodosol and contained 91.7\% sand, $5.2 \%$ silt, and $3.1 \%$ clay particles. In early May of 2012 a small-scale field experiment was established with

Contact address: Assoc. prof. Eugene Balashov, PhD, Agrophysical Research Institute, Department of Soil Physics, Physical Chemistry and Biophysics, 14 Grazhdansky prospekt, St. Petersburg, 195220, Russia, e-mail: Eugene Balashov@yahoo.co.uk 
forty bottomless plastic buckets $(30 \mathrm{~cm}$ in height and $30 \mathrm{~cm}$ in diameter). Empty buckets were dug into the 0-25 cm plough layers at an area of $5 \mathrm{~m}^{2}$. Afterwards, samples of soils with medium (MQ) and high (HQ) quality (resulting from different soil management for the previous ten years) collected from the $0-25 \mathrm{~cm}$ layers were placed in the buckets at a bulk density of $1.2 \mathrm{~g} . \mathrm{cm}^{-3}$. The experiment included four treatments: control (no biochar, no $\mathrm{N}$-fertilizer), biochar $\left(12\right.$ t.ha $\left.^{-1}\right)$, N-fertilizer $\left(90\right.$ kg.ha $\left.{ }^{-1} . \mathrm{N}\right)$ and biochar $\left(12\right.$ t.ha $\left.^{-1}\right)+$ $\mathrm{N}$-fertilizer $\left(90 \mathrm{~kg} \mathrm{ha}^{-1} \cdot \mathrm{N}\right)$. The mineral $\mathrm{N}$-fertilizer used in the experiment was ammonium nitrate $\left(\mathrm{NH}_{4} \mathrm{NO}_{3}\right)$. There were five randomized replicates of each treatment for the $\mathrm{HQ}$ and MQ soils.

During growing seasons (May - September) of 2012 2014 the average air temperature was $14.3^{\circ} \mathrm{C}, 15.5^{\circ} \mathrm{C}$, and $14.9^{\circ} \mathrm{C}$, respectively, while the total amount of precipitation for the periods reached $630 \mathrm{~mm}, 465 \mathrm{~mm}$ and $320 \mathrm{~mm}$, respectively.

The MQ soil had acidic reaction $\left(\mathrm{pH}_{\mathrm{KCl}} 5.6\right)$, contained 18.0 g.C. kg ${ }^{-1}$ soil of soil organic matter (SOM), 1.6 g.N. kg soil of total $\mathrm{N}$ and 10.5 mg. N. kg ${ }^{-1}$ soil of mineral nitrogen. The HQ soil had neutral reaction $\left(\mathrm{pH}_{\mathrm{KCl}} 6.5\right)$ and contained 23.0 g.C. kg ${ }^{-1}$ soil of SOM, 1.9 g.N. kg ${ }^{-1}$ soil of total $\mathrm{N}$ and 47.7 mg.N. $\mathrm{kg}^{-1}$ soil of mineral nitrogen. Spring barley (Hordeum vulgare L.) was grown on the experimental plot in 2012 and perennial grasses (Phleum pratense L. and Trifolium pratense L.) - in 2013 and 2014. The $\mathrm{N}$ fertilizers and biochar were mixed with topsoil layers $(0-10 \mathrm{~cm})$ at the beginning of the experiment. A particle size of the incorporated biochar was 2-5 $\mathrm{mm}$. A fresh slow pyrolysis biochar was produced from small birch logs and branches in a controlled kiln at temperature of the pyrolysis of $650{ }^{\circ} \mathrm{C}$. Afterwards, the produced biochar (or non aged biochar) was oxidated in air conditions during one year. Chemical and physical properties of the non aged biochar were: total carbon content -825.5 g.C.kg ${ }^{-1}$, total $\mathrm{N}$ content -5.7 g.N.kg ${ }^{-1}, \mathrm{C} / \mathrm{N}$ ratio $-145, \mathrm{pH}_{\mathrm{H}_{2} \mathrm{O}}-7.0$, moisture content $-1.92 \%$ and ash content $-0.23 \%$. All the soil chemical and physical analyses were conducted by standard and traditional methods used in Russian soil science laboratories (Rastvorova et al., 1995).

In September of 2014, bulk MQ and HQ soil samples and biochar particles of 2-5 mm were collected from experimental plots with the control and biochar $\left(12\right.$ t.ha $\left.^{-1}\right)$ treatments. Air-dried soil samples were sieved through sieves of $3 \mathrm{~mm}$ and $5 \mathrm{~mm}$ to receive $3-5 \mathrm{~mm}$ soil fraction. Afterwards, these soil and biochar samples were stored for 5 years in hermetically sealed plastic vessels without access of oxygen at a room temperature of $20{ }^{\circ} \mathrm{C}$. It was assumed that these storage conditions could not result in any significant changes in the surface and physicochemical properties of the soil and biochar samples. The 3-5 mm size fractions of the soils and $2-5 \mathrm{~mm}$ size fractions of the biochar were used in our study.

Before the beginning of water vapor adsorptiondesorption measurements, all the plastic vessels were opened and all the soil and biochar samples were kept at a room temperature of $23.5^{\circ} \mathrm{C}$ and at an ambient laboratory atmosphere for one day to achieve an equilibrium content of hygroscopic water in the samples.

A dewpoint potentiameter (WP4-T, Decagon Devices, Inc., Pullman, WA, USA) was used to measure water potentials of the biochar and soil samples. Weight of biochar and soil samples was equal to $0.5 \mathrm{~g}$ and $2 \mathrm{~g}$, respectively. Five cycles of water vapor adsorption-desorption processes were carried out for the biochar and soil samples. During the $24 \mathrm{~h}$ adsorption process, the samples were saturated by water vapor in plastic vapor-tight vessels $\left(100 \mathrm{~cm}^{3}\right)$ over distilled water at $95-98 \%$ relative air humidity $\left(p / p_{o}\right)$ up to equilibrium maximum hygroscopic water content $\left(\mathrm{HWC}_{\max }\right)$. The measurements of water potentials and weights of the biochar and soil samples were carried out immediately after the end of the adsorption process. Afterwards, the desorption process included several subsequent measurements (every 30-60 minutes) of the water potentials and weights of biochar and soil samples until the samples were reaching equilibrium minimum hygroscopic water content $\left(\mathrm{HWC}_{\min }\right)$. After the end of the fifth cycle of adsorption-desorption process the biochar and soil samples were dried at $105^{\circ} \mathrm{C}$ and their moisture content was calculated. These data are presented in the hygroscopic water retention curves. The same sample (both for the biochar and the soils) was used in the five subsequent cycles of water vapor adsorptiondesorption processes.

Means and standard deviations were calculated for $H W C_{\min }$ and $H W C_{\max }$ of all the studied samples after the five cycles of water vapor adsorption-desorption processes. The results were subjected to the analysis of variance (one-way ANOVA) at $P \leq 0.05$. Strength of associations between sets of the parameters was assessed with Pearson correlation coefficients at $P \leq 0.05$.

\section{Results and discussion}

Fresh biochar is usually hydrophobic, but if its surface was oxidized by air or water molecules, biochar becomes more hydrophilic and accessible to stronger chemical interactions with soil minerals with subsequent physical occlusion in organo-mineral fractions (Lehmann et al., 2011).

Our results showed that the highest values of $\mathrm{HWC}_{\max }$ were observed in the non aged biochar $-14.07 \% \pm 0.37 \%$ (of weight). The ageing of biochar in the $\mathrm{MQ}$ and $\mathrm{HQ}$ soils resulted in a decrease of $\mathrm{HWC}_{\max }$ to $10.33 \% \pm 0.17 \%$ and $10.52 \% \pm 0.65 \%$, respectively. There were no significant differences between the $\mathrm{HWC}_{\max }$ of the aged biochars from the $M Q$ and $H Q$ soils, but the differences between the values of $\mathrm{HWC}_{\max }$ in the non aged biochar and the two aged biochars were statistically significant $(P<0.001)$.

The $\mathrm{HWC}_{\max }$ of $\mathrm{MQ}$ and $\mathrm{HQ}$ soils showed lower values $(2.86 \% \pm 0.14 \%$ and $4.39 \% \pm 0.89 \%$, respectively) than those of the aged biochars. Because the clay content and mineralogical composition of the $\mathrm{MQ}$ and $\mathrm{HQ}$ soils were similar, the significant $(P<0.05)$ differences in the $H W C_{\max }$ of $\mathrm{MQ}$ and $\mathrm{HQ}$ soils could be attributed to those in the SOM content (18.0 g.C.kg ${ }^{-1}$ soil and 23.0 g.C.kg ${ }^{-1}$ soil, respectively). The higher hydrophilicity of HQ soil did not result in any significant changes in the hydrophilicity of the aged biochar compared to that of the aged biochar from MQ soil.

After the end of the water vapor adsorption processes values of the biochars and soils water potentials relatively well reflected the above-mentioned differences in their $\mathrm{HWC}_{\max }$. The water potentials were equal to: $-5.5 \mathrm{MPa} \pm$ 
-1.3 MPa (non aged biochar), $-9.8 \mathrm{MPa} \pm-1.4 \mathrm{MPa}$ (aged biochar from $\mathrm{HQ}$ soil), $-7.2 \mathrm{MPa} \pm-0.8 \mathrm{MPa}$ (aged biochar from MQ soil), $-5.2 \mathrm{MPa} \pm-0.6 \mathrm{MPa}$ (HQ soil) and $-8.1 \mathrm{MPa} \pm$ $-2.3 \mathrm{MPa}$ (MQ soil). Significant differences in water potentials were observed between the non aged biochar and aged biochar from MQ soil $(P<0.05)$. Water potentials of the MQ and $\mathrm{HQ}$ soils also demonstrated significant differences in water potential $(P<0.001)$. The ageing of biochars in the soils led to a stronger association of hygroscopic water with biochar surfaces at $\mathrm{HWC}_{\max }$ compared to non aged biochar.

During the adsorption process at low $p / p_{o}$ water molecules form bonds with OFGs and previously adsorbed water molecules, and then form separate and convex water clusters on an adsorbent surface. If $\mathrm{p} / \mathrm{p} 0$ increases up to high values ( $>50 \%)$, such water clusters grow and fill in micropores of an adsorbent. At $p / p_{o}$ between the formation of the initial water clusters and the condensation, water clusters in micropores may grow and coalesce as a result of hydrogen bonding formation between them. The adsorption rates are fastest at low air humidity and are slowest at condensation. In mesopores (at $p / p_{0} 80-90 \%$ ) larger clusters form bridges, and the growth of clusters prior to bridging requires a higher chemical potential and therefore, water adsorption occurs progressively from smaller to larger pores (Liu et al., 2017).

The $\mathrm{HWC}_{\max }$ depends on the density of OFGs and the volume and size of pores available for water vapor adsorption at a given $\mathrm{p} / \mathrm{p} 0$. Among the OFGs, carboxyl groups demonstrate the highest affinity towards water molecules (Brennan et al., 2001). Water molecules tend to cluster around carboxyl groups, regardless of the presence of carbonyl or hydroxyl functional groups. At the pore filling stage the rates of water uptake are inversely proportional to the pore sizes.

The 3-year ageing of biochar in both soils had probably caused a decrease in the density of OFGs on the biochar surface and in the available volume of pores because of their blocking by clay particles. Mineralogical composition of the soil clay particles was mainly presented by quartz and potassium feldspar with a low affinity to water molecules (Boitsova et al., 2015).
The results of the studies of the water desorption process from $\mathrm{HWC}_{\max }$ to $\mathrm{HWC}_{\text {min }}$ demonstrated a higher water retention of the HQ soil than that of the MQ soil (Figure 1).

There were significant $(P<0.001)$ differences between hygroscopic water retention curves of the $\mathrm{HQ}$ and $\mathrm{MQ}$ soils. If the whole desorption process was considered, mean values of corresponding water potentials of the $\mathrm{HQ}(-98.1 \mathrm{MPa})$ and MQ (-96.7 MPa) soils showed only insignificant differences. Pearson correlation coefficient between hygroscopic water retention curves of the $\mathrm{HQ}$ and $\mathrm{MQ}$ soil was equal to 0.98 $(P<0.001)$. The results showed that desorption processes of hygroscopic water from both soils probably occurred from pores of similar size. The significant differences between hygroscopic water retention curves could be mainly induced by the differences in hydrophilic SOM contents between the $\mathrm{HQ}$ and $\mathrm{MQ}$ soils. There were significant $(P<0.05)$ differences between mean values of $\mathrm{HWC}_{\text {min }}$ of the $\mathrm{HQ}(1.42 \% \pm 0.17 \%)$ and MQ $(1.16 \% \pm 0.03 \%)$ soils. The mean values of $\mathrm{HWC}_{\min }$ corresponded to mean water potentials of $-190.5 \mathrm{MPa} \pm$ -13.3 MPa for $\mathrm{HQ}$ soil and -186.0 MPa $\pm-14.4 \mathrm{MPa}$ for MQ soil. According to Do et al. (2009), the desorption process of hygroscopic water in activated carbons resulted from the decomposition of the larger (superclusters) to smaller clusters before they are desorbed from the micropores. A magnitude of desorption process depends on a number of superclusters in the pores and on their volumes and sizes.

The magnitude of decreasing water potentials was smaller at the initial stage (from $\mathrm{HWC}_{\max }$ ) of desorption process for the non aged biochar than for the aged biochar. For instance, during the first 30 min of desorption a decrease in water potentials reached $-70 \mathrm{MPa}$ for non aged biochar and $-130 \mathrm{MPa}$ for aged biochars. Further temporal stages of desorption (down to $\mathrm{HWC}_{\min }$ ) were characterized by the lower magnitude of decrease in water potentials of the aged biochars compared to non aged biochar. The differences in the magnitudes of decreasing water potentials could be attributed to higher volumes of emptying pores in non aged biochar.

During the desorption process mean values of corresponding water potentials were equal to $-109.8 \mathrm{MPa}$

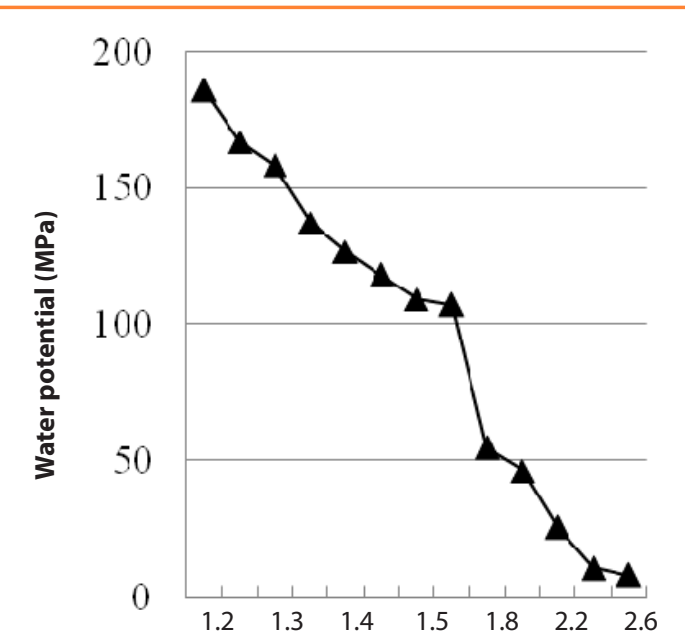

(a)

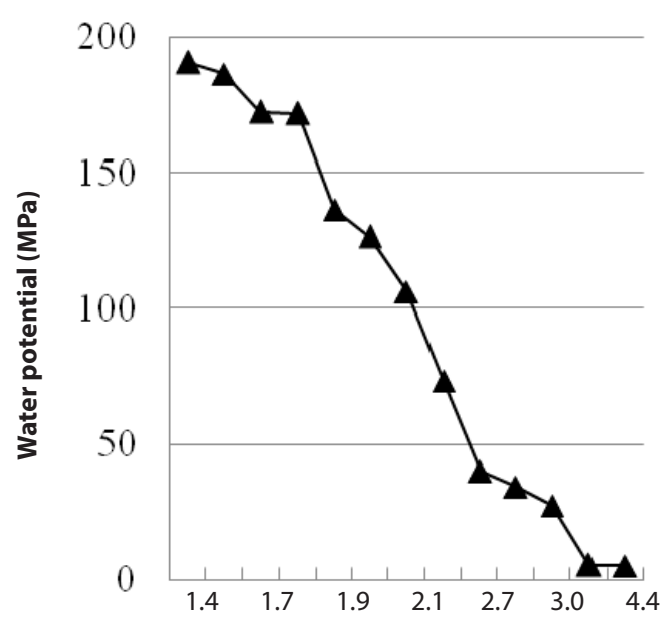

(b)

Hydroscopic water content (\% of weight)

Figure 1 Hygroscopic water retention curves of sandy Spodosols with (a) medium and (b) high quality 


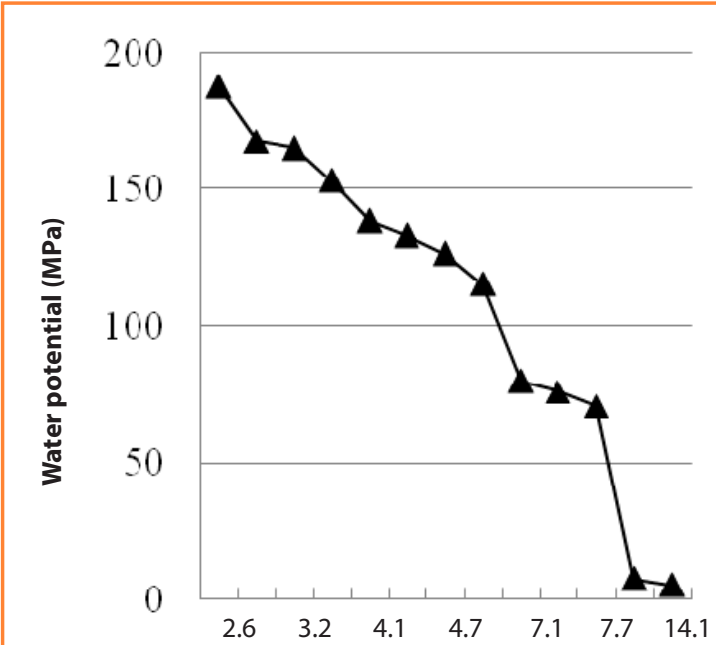

(a)

Hydroscopic water content (\% of weight)

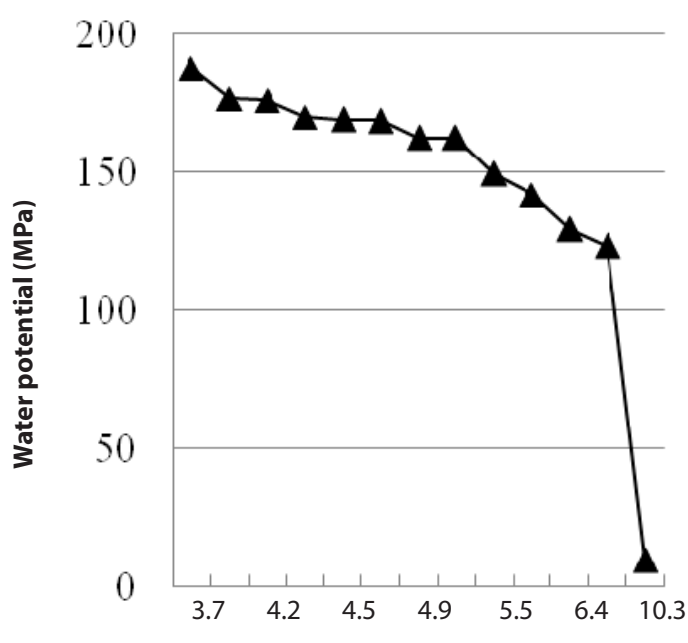

(b)

Hydroscopic water content (\% of weight)

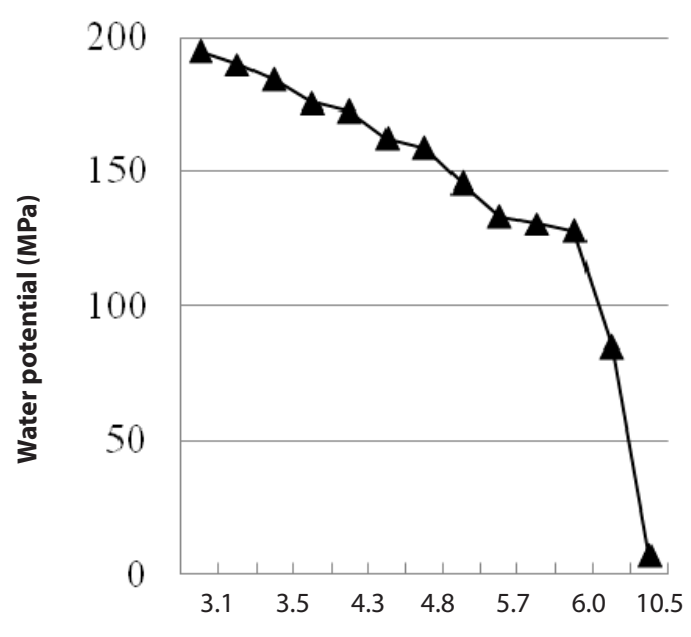

(c)

Hydroscopic water content (\% of weight)

Figure 2 Hygroscopic water retention curves of (a) non aged biochar, (b) aged biochar from soil with high quality and (c) medium quality (non aged biochar), $-148.3 \mathrm{MPa}$ (aged biochar from $\mathrm{HQ}$ soil) and $-143.6 \mathrm{MPa}$ (aged biochar from MQ soil). There were significant differences in the mean water potentials of the non aged biochar and the aged biochars from the HQ soil $(P<0.01)$ and MQ soil $(P<0.001)$.

There were insignificant differences between the water retention curves of the studied biochars during the desorption processes. However two aged biochars retained more hygroscopic water than non aged biochar at the same corresponding water potentials (Fig. 2). Desorption rates of hygroscopic water were lower from the aged biochars than from the non aged biochar which probably had higher volumes of pores. Therefore a decrease in the same amount of desorbed hygroscopic water corresponded to a higher difference in water potentials of the non aged biochar compared to two aged biochars.

Mean values of $\mathrm{HWC}_{\min }$ and corresponding water potentials for the biochars were equal to: $2.61 \% \pm 0.08 \%$ $(-187.6 \mathrm{MPa} \pm-4.4 \mathrm{MPa})$ for non aged biochar, 3.75\% $\pm 0.09 \%$ $(-187.7 \mathrm{MPa} \pm-1.8 \mathrm{MPa})$ for aged biochar from $\mathrm{HQ}$ soil and $3.14 \% \pm 0.12 \%(-194.8 \mathrm{MPa} \pm-5.2 \mathrm{MPa})$ for aged biochar from MQ soil. There were significant differences $(P<0.001)$ in $\mathrm{HWC}_{\text {min }}$ between all biochars. Significant differences in water potentials were observed for the aged biochar from MQ soil versus HQ soil $(P<0.05)$ and versus the non aged biochar $(P<0.05)$.

By subtracting the values of $\mathrm{HWC}_{\min }$ from $\mathrm{HWC}_{\max }$ the absolute amounts of adsorbed water can be calculated for all the biochars and soils during their saturation at high values of $p / p_{o}$. These amount of adsorbed water ranged in the following decreasing order: $114.6 \mathrm{~g} . \mathrm{kg}^{-1} \pm 3.2 \mathrm{~g} . \mathrm{kg}^{-1}$ (non aged biochar), 73.7 g. $\mathrm{kg}^{-1} \pm 5.9 \mathrm{~g} . \mathrm{kg}^{-1}$ (aged biochar

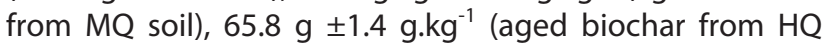
soil), $29.7 \mathrm{~g} . \mathrm{kg}^{-1} \pm 8.7 \mathrm{~g} . \mathrm{kg}^{-1}$ (HQ soil), $14.0 \mathrm{~g} . \mathrm{kg}^{-1} \pm 1.6 \mathrm{~g} . \mathrm{kg}^{-1}$ (MQ soil). These data showed that the improvement of soil quality had significantly $(P<0.05)$ promoted the affinity of the soil to water vapor, probably as a result of increase in the content of hydrophilic SOM. The significant changes in the water vapor adsorption by the $\mathrm{HQ}$ soil did not contribute to the similar changes in the aged biochar. In opposite, as compared to the non aged biochar, both aged biochars demonstrated a significant decrease $(P<0.001)$ in the absolute amounts of adsorbed water.

\section{Conclusions}

The 3-year ageing of biochar in the soils with medium and high quality caused a significant $(P<0.05$ and $P<0.01)$ decrease in their maximum hygroscopic water content as compared to that of the non aged biochar. A significantly $(P<0.05)$ higher hydrophilicity of the soil with high quality compared to the soil with medium quality did not contribute to any significant increase in the soil maximum hygroscopic water content. The ageing of biochar in both soils could cause a decrease in the density of the biochar oxygen-containing functional groups and in the available volume of pores as a result of their blocking by clay particles with low affinity to water vapor.

The 3-year ageing of biochars in both soils resulted in a significant $(P<0.001)$ increase in their minimum hygroscopic water content compared to that of the non aged biochar after finishing the process of hygroscopic 
water desorption. The desorption rates of hygroscopic water were lower from two aged biochars than those from the non aged biochar which probably had higher volumes of microand mesopores.

\section{Acknowledgement}

This study was supported by the Russian Foundation for Basic Research (grant No. 19-016-00038-A).

\section{References}

BOITSOVA, L. - ZINCZUK, E. - NEPRIMEROVA, S. - BALASHOV, E. 2015. Distribution of total and clay-associated organic matter in profiles of arable loamy sand Spodosol. In Folia Oecologica, vol. 42 2015, no. 1, pp. 1-9. ISSN 1336-5266.

BRENNAN, J. K. - BANDOSZ, T. J. - THOMSON, K. T. - GUBBINS, K. E. 2001. Water in porous carbons. In Colloids and Surfaces $A$ Physicochemical and engineering aspects, vol. 187, 2001, pp. 539568. ISSN 0927-7757.

DO, D. D. - JUNPIROM, S. - DO, H. D. 2009. A new adsorptiondesorption model for water adsorption in activated carbon. In Carbon, vol. 47, 2009, no. 6, pp. 1466-1473. ISSN 0016-7061.

EL-NAGGAR, A. - LEE, S. S. - RINKLEBE, J. - FAROOQ, M. - SONG, H. - SARMAH, A. K. - ZIMMERMAN, A. R. - AHMAD, M. - SHAHEEN, S.M. - OK, Y. S. 2019. Biochar application to low fertility soils: A review of current status, and future prospects. In Geoderma, vol. 337, 2019, pp. 536-554. ISSN 0016-7061.
HORÁK, J. 2015. Testing biochar as a possible way to ameliorate slightly acidic soil at the research field located in the Danubian lowland. In Acta Horticulturae et Regiotecturae, vol. 18, 2015, no. 1 , pp. 20-24. ISSN 1338-5259.

KEILUWEIT, M. - NICO, P. S. - JOHNSON, M. G. - KLEBER, M. 2010. Dynamic molecular structure of plant biomass-derived black carbon (biochar). In Environmental Science \& Technology, vol. 44, 2010, no. 4, pp. 1247-1253. ISSN 0013-936X.

LEHMANN, J. - RILLIG, M. C. - THIES, J. - MASIELLO, C. A. - HOKADAY, W. C. - CROWLEY, D. 2011. Biochar effects on soil biota-a review. In Soil Biology and Biochemistry, vol. 43, 2011, no. 9, pp. 1812-1836. ISSN 0038-0717.

LIU, L. - TAN, S. J. - HORIKAWA, T. - DO, D. D. - NICHOLSON, D. LIU, J. 2017. Water adsorption on carbon - A review. In Advances in Colloid and Interface Science, vol. 250, 2017, pp. 64-78. ISSN 0001-8686.

MARSHALL, J. - MUHLACK, R. - MORTON, B. J. - DUNNIGAN, L. CHITTLEBOROUGH, D. - KWONG, C. W. 2019. Pyrolysis temperature effects on biochar - Water interactions and application for improved water holding capacity in vineyard soils. In Soil Systems, vol. 3, 2019, no. 2, p. 27. ISSN 2571-8789.

RASTVOROVA, O. G. - ANDREEV, A. P. - GAGARINA, E. I. KASATKINA, G. A. - FYEDOROVA, N. N. 1995. Chemical analysis of soils. St. Petersburg: St. Petersburg University Publishing, 1995, 264 p. (in Russian). 